\title{
Grade 11 Learners' Misconceptions and Inability to Apply Basic Algebra Operations: The Case of Selected Secondary Schools in Chilanga District, Zambia
}

\author{
Jane Sikombe ${ }^{1 *} \quad$ Rex L Sampa $^{2} \quad$ Allan Musonda $^{3}$ \\ 1.Ministry of General Education, Mount Makulu Secondary School, Lusaka, Zambia \\ 2.School of Humanities \& Social Sciences, Copperbelt University, Kitwe, Zambia \\ 3.School of Mathematics \& Natural Sciences, Copperbelt University, Kitwe, Zambia
}

\begin{abstract}
The aim of the study was to assess and establish learners' misconception and inability to apply simplification of algebraic expressions and solving linear equations in Chilanga District. The study sought to determine the possible causes of learners' inability to apply simplification of algebraic expressions and solving linear equations in relation to identification of inverses in senior secondary school. A case study research design was employed to achieve the objectives and a sample of 100 grade 11 learners and 10 mathematics teachers from school A and school B participated in the study. To collect data the instruments used were a questionnaire, tests and interviews. Quantitative data was analyzed using an Excel statistical package while the reliability of the instruments was tested using Cronbach's alpha $(\alpha)$. The main findings the research study established are that learners misused and misapplied addition, subtraction, multiplication and division when simplifying algebraic expressions and forming linear equations. Learners also met challenges when it came to solving linear equations because they lacked knowledge of additive and multiplicative inverses. The main conclusions drawn from this research study are that misconceptions learners experience in algebra are attributed to the lack of conceptual knowledge and understanding and these misconceptions occurred frequently in learners.
\end{abstract}

Keywords: Level of Education (Grade 11), Learners' Misconception, Inability to apply basic Algebra Operation. DOI: $10.7176 / \mathrm{JEP} / 12-33-11$

Publication date: November $30^{\text {th }} 2021$

\subsection{INTRODUCTION}

An understanding of symbols and operations is very important to the introduction of school algebra. Symbols in algebra are related to notation and expression, usually letters (variables) and signs of operations. Letters in algebra are called variables and many learners are of the view that letters represent numbers, thus school algebra is regarded as generalized arithmetic (Mwape, 2016). In word problems learners experience challenges to form algebraic expressions and linear equations because of poor understanding of operations (addition, subtraction, multiplication and division).

Knowledge of algebra is vital throughout life. This knowledge affects the decision made in many areas such as in finance, technology and daily life. The demand for algebra at higher levels of education is also on the rise. Many students struggle learning mathematics in grades 8 and other grades because there understanding of algebra is not deep. This claim is supported by Maguire (2012), who has revealed that lower grade students often misconceive equivalence as an operation rather than a relation, and that students also form various misconceptions of literal symbols.

According to Gunawadena (2011), the main reason for learners' misconceptions in algebra is lack of understanding of the basic concepts of the variables in different contexts. Misconceptions in this context means an idea that is wrong because it has been based on a failure to understand concepts of basic operation of algebra. Thus, preventing algebraic misconceptions for instance, when simplifying algebraic expressions and solving linear equations dealing with addition, subtraction, multiplication and division may be necessary for increasing learners' success in algebra.

Learners' misconceptions should be identified and prevented because they result into misapplication and misuse of basic operations of algebraic expressions. These expressions are usually misapplied in many learning contexts. For example, where the operation of division is supposed to be used, another operation such as multiplication or minus may be wrongly used. This misapplication or misuse of operations is as a result of misconceptions in algebra and results in misleading answers. The example of the foregoing can be seen in business when selling goods; people may want to know how much each unit of an item is costing in order to come up with a selling price. Instead of starting with a division operation to know the cost price per unit, an addition operation is used first and end with division which is quite misleading. This is a cause for concern because the topic on integers forms a fundamental background needed for success in algebra and higher-level mathematics and everyday life.

During the researcher's secondary school teaching career, it was observed that many learners have problems 
concerning basic operations of algebra such as, identification of variables, simplification of algebraic expressions and forming linear equations. They have problems in adding, subtracting, multiplying and dividing algebraic expressions and are unable to apply the concepts in other topics, other subjects and other fields. Discussions with fellow teachers have confirmed that they too have observed the same challenge which is persistent. Misconceptions in mathematics learning are a common occurrence across different cohorts of learners (Mwape, 2016). This study provides an insight into the misconceptions and remedial work that occur when teaching challenging topics such as algebra in mathematics.

\subsection{Theoretical /Conceptual Framework}

The aim of the study was to investigate learners' misconceptions and inability to apply concepts of basic operations in algebra. Constructivism seemed consistent and appropriate for studying learners' understanding and thinking. Hence, the study is informed by the theory of constructivism. A constructivist perspective on learning (Piaget, 1970; Skemp, 1979; Ausebel, 1976) assumes that concepts are not taken directly from experience, but that a person's ability to learn from what he/she learns from an experience. Observation is driven by theory, so the quality of observation is determined by the quality of the pre-existing theory. Rather, it arises from interaction between experience and learners' current knowledge structures. This research took the constructivist approach because when it comes to the idea of a perfect classroom, it is believed that actively engaging the learners in learning is the most productive means of teaching.

Piaget defined intelligence as the ability to adapt to the environment. Adaptation takes place through assimilation and accommodation, with two processes of interacting throughout life in different ways, according to the stages of mental development. In assimilation, the individual absorbs new information, fitting features of the environment into cognitive structures. In accommodation, the individual modifies those internal cognitive structures to conform to the new information and meet the demands of the environment. In that way, a balance is maintained through equilibrium, as the individual organizes the demands of the environment in terms of previously existing cognitive structures.

Providing various mathematical representations acknowledges the uniqueness of learners and provides multiple paths for making ideas meaningful. Giving opportunities for learners to present mathematical solutions in multiple ways (symbols, graphs, tables and words) is one tool for cognitive development in this stage. Eggen and Kauchak (2000) noted that while a specific way of presenting an idea is meaningful to some learners a different presentation might be more meaningful to others. This study used the fourth stages of Piaget's child development to assess learners' misconceptions and ability to apply basic algebraic operations. The constructivist perspective, developed as a part of Piaget, asserts that conceptual knowledge cannot be transferred from one person to another but constructed by the individual learner (Piaget, 1970).

\subsection{Purpose of the Study}

The purpose of this study was to investigate learners' misconceptions and inability to apply addition, subtraction, multiplication and division when identifying variables, simplifying algebraic expressions and solving linear equations at senior secondary school. In this context, inability meant being unable to put to use the basic rules of the algebraic concepts. The study explored misconceptions in learners when solving problems related to identification of variables, simplification of algebraic expressions and solving of linear equations. Furthermore, explore the possible causes of learners' inability to apply addition, subtraction, multiplication, division and it will also assess learners' problems in solving linear equations in two variables using elimination method were explored. The study was guided by three research questions:

- What are the possible causes of learners' inability to apply simplification of algebraic expressions and solving linear equations in relation to identification of inverses in senior secondary school?

- What are the problems observed in learners when solving and reasoning on algebraic concepts?

- How can the learners' problems in solving and reasoning on algebraic concepts be approached?

\subsection{METHOD}

The study sought to investigate learners' misconceptions and inability to apply concepts of three basic operations of algebra that is, identification of variables, simplification of algebraic expressions and linear equations at grade eleven level in two selected secondary schools of Chilanga district, Zambia. Therefore, a case study design was used as this design increased the understanding of the problem that was identified. The instruments used to collect data were a questionnaire, tests and interviews. Quantitative data was analyzed using an Excel statistical package while the reliability of the instruments was tested using Cronbach's alpha $(\alpha)$. The use of three instruments was a means of ensuring that accurate results were brought out. The study applied both quantitative and qualitative approaches. 


\subsection{Research Participants}

The participants were drawn from the population of grade 11 learners of two selected secondary schools in Chilanga district. The researcher targeted learners and teachers from school A with a population of 230 grade 11 learners as well as 5 mathematics teachers and School B with a population of 210 grade 11 learners and 5 mathematics teachers. In this research the lottery method was used to come up with 100 participates as the sample. The lottery method was one of the examples of random sampling in which the researcher gave each member of the population a number. The numbers 1-230 were written on pieces of paper and put in a box and shaken to allow every grade 11 learner to pick a number with replacement at School A. The ones who picked numbers 1-50 participated in the study. Similarly, numbers 1-210 were written on pieces of paper and put in the box as well as shaken and allowed every grade 11 learner to pick a number with replacement at School B and those who picked 1-50 participated in this research. The researcher gathered 100 participants to write the assessment test in algebra and 5 participants to be interviewed based on the way the learner answered the test questions as well as 5-teachers to answer the questionnaire.

\subsection{Research Instruments}

The instruments used to collect data in this study were a questionnaire, tests and interview schedules.

\subsection{Reliability and Validity of the Instruments}

According to Cohen et al. (2000) reliability refers to the degree of confidence that can be placed in the results and the data, and this is usually an issue of statistical calculation. Validity, on the other hand, refers to the extent to which an instrument measures what it is supposed to measure. In this study, in order to ensure validity of the instruments the researcher conducted a pilot study. The results of the pilot study were compared to the results of the main study for correlation

A pilot-test was conducted for 23 learners who wrote an assessment in algebra to investigate learners' misconceptions and ability to apply addition, subtraction, multiplication and division when identifying variables, simplifying algebraic expressions and solving linear equations. From the data of the test, the researcher determined the reliability of the instrument by calculating the Cronbach Alph $(\alpha)$ of the data.

\subsection{Research Procedure}

A self-designed test, based on algebra which depended on the research questions, was used to collect data from learners. The first part of the research comprised of a test with a total of 50 marks, which was used to analyse grade 11 learners' misconceptions and ability to apply addition, subtraction, multiplication and division when identifying variables, simplification of algebraic expressions and solving linear equations learnt in the senior secondary school. The purpose of a test was to give valid information on students' abilities and knowledge. Hence, the success of teaching and learning can be seen in the test's results

The second part consisted of interview schedule and Teachers' questionnaire which focused on examining the causes of learners' misconceptions and inability to apply addition, subtraction, multiplication and division when simplifying algebraic expressions and solving linear equations displayed by grade 11 learners as well as how to avoid the misconceptions when applying basic algebra concepts in everyday life. Teachers answered eight questions on the questionnaire based on the research questions.

\subsection{RESULTS AND FINDINGS}

Research data analysis involved the process of making sense out of data and included consolidating, reducing and interpreting how learners and teachers have answered the test and questionnaire respectively as well as how the learners answered during the interview observed by the researcher.

Both quantitative and qualitative data were used in this case study in order to investigate learners' misconceptions and inability to apply addition, subtraction, multiplication and division at senior secondary school in a case study of Chilanga District. Misconceptions in learners when solving problems related to identification of variables, simplification of algebraic expressions and solving linear equations were explored. Possible causes of learners' inability to apply addition, subtraction, multiplication and division and when identifying variables, simplifying algebraic expressions and solving linear equations and assessed learners' problems in solving linear equations in two variables using elimination method were also explored.

The information collected from participants was analyzed using descriptive statistics (frequency, counter, percentages, tabulation) and coded as well as thematic content analysis. The presentation, interpretation and analysis of data followed the form of the research questions:

- What are the possible causes of learners' inability to apply simplification of algebraic expressions and solving linear equations in relation to identification of inverses in senior secondary school?

- What are the problems observed in learners when solving and reasoning on algebraic concepts?

- How can the learners' problems in solving and reasoning on algebraic concepts be approached? 
3.1 Findings regarding the quantitative (test and questionnaire) and qualitative (interview) investigations. The results and discussion of the findings focuses on learners' scripts when answering algebraic questions. These questions included identification of variables, simplification of expressions and solving linear equations. These results were guided by research objectives, the research questions and the results of the study using analysis of the learners' scripts.

\section{Misconceptions in Learners when solving problems related to identification of Variables, simplification} of algebraic expressions and solving of linear equations

Learners' misconceptions and inability to apply basic algebra operations (addition, subtraction, multiplication and division) were looked at as well as the causes of learners' inability to apply addition, subtraction, multiplication and division when identifying variables, simplifying algebraic expressions and solving linear equations.

Question 1 was based on algebraic variables and numerical values whereby learners were assessed on ability to identify and clarify the variables. The question addressed the problem of learner misconceptions and inability to apply addition, subtraction, multiplication when identifying variables, simplifying algebraic expressions and solving linear equations as well as find the causes and remedies to the problem. According to the theory of constructivism by Piaget (1970), learners in grade 11 are in the formal operational stage and they are expected to be able to identify and clarify between variables and numerical values. The learners were given to respond to the equation $6 a+3 x=7 y$.

The findings revealed that learners have misconceptions between variables and numerical values. About $89 \%$ of the learners have misconceptions in identifying variables and numerical values. This was also observed in the written test and teachers' questionnaire.

Under algebraic expressions, a good number of pupils had misconceptions when multiplying expressions involving directed numbers. Furthermore, findings revealed that about $97 \%$ of learners had misconceptions in application of algebraic operations (addition, subtraction, multiplication and division) were displayed when simplifying and formation of algebraic expressions.

The lack of understanding of a variable as a generalized number is a misconception observed in a few questions during the interview with a learner who did not realize that the two variables $\mathrm{x}$ and $y$ can take more than one value in the equation. Further, the particular learner focused only on the domain of positive numbers to decide which variable was larger than the other and disregarded a zero as a value for substitution.

As regards incomplete simplification, an answer was categorized incomplete if a learner terminated the simplification of an algebraic expression before the accepted answer. In this situation a learner would start the problem and proceed with one or two steps and abruptly terminates the process before reaching the final answer. Through the written test and the interviews conducted with the learners it was observed that probably there were two reasons for this. Firstly, it could be that learners did not know how to proceed with the problem. They misapplied and misused the operations addition, subtraction, multiplication and division. Secondly, learners could have thought they had reached the final answer. For the incomplete answers, further simplification was possible to reach the desirable solution.

This study also revealed that there were was over simplification of algebraic expressions by some participants. The findings have revealed that fifty three percent $(53 \%)$ of the learners perceived an algebraic expression as an equation which led them to over simplification of the expression.

Causes of Learners' Inability to apply addition, subtraction, multiplication and division when simplifying Algebraic expressions and solving linear equations

When it came to examining learner's ability to apply addition, subtraction, multiplication and division in simplification of algebraic expressions as well as formation of linear equations and solve, it was revealed that $97 \%$ of the learners got the question wrong. Learners multiplied $-2 \mathrm{a}$ and $(+\mathrm{y})=+$ which was supposed to be $(-)$ $\mathrm{x}(+)=-$. Many $(83 \%)$ of the participants had misconceptions on directed numbers

When learners were tested on applying the formula for area of a rectangle, learners instead wrote the area of a right-angled triangle as $\mathrm{A}=\frac{b h}{2}$ which was supposed to be $\mathrm{A}=1 \times \mathrm{b}=\mathrm{lb}$. Hence learners were unable to write an expression for area in terms of $\mathrm{L}$ due to misapplication and misuse of multiplication and division. It was also observed that learners did not mention the expression which was formed.

Lack of algebraic understanding of basic operations, and poor foundation knowledge especially on directed numbers appear to be the major causes of learners being unable to apply addition, subtraction, multiplication and division in identification of variables, simplifications of expressions and solving of linear equations. Participants seemed not to know how to apply and perform mathematical operations namely: addition, subtraction, multiplication and division

On question 3 findings were that learners had problems to make expressions and solve the equations. The question was a real-life problem in the application of algebraic operation. Learners faced challenges solving the variables as they could not identify the additive and multiplicative inverses. 
Table 1: Total number of participants by gender

\begin{tabular}{|l|l|l|l|}
\hline & Pupils & Teachers & Managers/Administrators: \\
\hline Males & 58 & 07 & 09 \\
\hline Females & 42 & 03 & 09 \\
\hline Total & 100 & 10 & 18 \\
\hline
\end{tabular}

Table 2: School A number of participants by gender

\begin{tabular}{|l|l|l|l|}
\hline & Pupils & Teachers & Managers/Administrators: \\
\hline Males & 35 & 04 & 04 \\
\hline Females & 15 & 01 & 05 \\
\hline Total & 50 & 05 & 09 \\
\hline
\end{tabular}

Table 3: School B number of participants by gender

\begin{tabular}{|l|l|l|l|}
\hline & Pupils & Teachers & Managers/Administrators: \\
\hline Males & 23 & 03 & 05 \\
\hline Females & 27 & 02 & 04 \\
\hline Total & 50 & 05 & 09 \\
\hline
\end{tabular}

\subsection{DISCUSSION AND CONCLUSION}

The discussion and conclusion are framed in line with the research questions of the study. Therefore, question 1 will be presented first, followed by question 2 and question 3 .

Question 1 addressed the problem of learner misconceptions and inability to apply addition, subtraction, multiplication when identifying variables, simplifying algebraic expressions and solving linear equations as well as find the causes and remedies to the problem. According to the theory of constructivism by Piaget (1970), learners in grade 11 are in the formal operational stage and they are expected to be able to identify and clarify between variables and numerical values. The learners were given to respond to the equation $6 a+3 x=7 y$. As revealed in the results section, many $(89 \%)$ of the participants had misconceptions in identifying variables and numerical values. Misconceptions could be as a result of lack of understanding of basic concepts of algebra. As Gunawadadena (2011), argued the main reasons for misconceptions is lack of understanding of basic algebra variables in different context. This situation may be remedied by the use of various teaching approaches such as those which are learner centered.

Question 2 asked about the problems observed in learners when solving and reasoning on algebraic concepts. As noted in the Results section about $97 \%$ of learners had misconceptions in application of algebraic operations addition, subtraction, multiplication and division. Misconceptions were as a result of lack of understanding of basic algebraic concepts. Apart from that it was also true that the participants lacked arithmetic skills. It has been claimed earlier that algebra is generalized arithmetic (Mwape, 2016). Thus, the research here contends that lack of a firm foundation in arithmetic skills may lead to misconceptions in algebra. This is supported by a number of researchers (Norton \& Irvin, 2007; Stacey \& Macgregor, 1999). They assert that poor arithmetic skills such as identification of additive and multiplicative inverses are the major factors that contribute to algebraic misconceptions when solving linear equations.

Question 3 considers how the learners' problems in solving and reasoning on algebraic concepts can be approached. As argued earlier it has been established that learners' problems in algebra can be attributed to the learners' lack of arithmetic skills. This was observed in the learners' lack of identification of additive and multiplicative inverses and how to use them in a given algebraic question. This may be overcome by the teacher providing opportunities for learners to familiarize them with the needed arithmetic skills. Familiarity with arithmetic skills of addition, subtraction, multiplication and division. Furthermore, understanding of arithmetic concepts such as additive inverses, multiplicative inverses. This will help learners connect the relationships observed in arithmetic to algebraic concepts.

One of the important concepts in algebra is that of a variable. Just as algebra is referred to as generalized arithmetic, a variable is a generalized number. This suggests that it can stand in place of different numbers at different times and in different contexts. For example; $x+3=5, x-3=5, \frac{x}{3}=5$ and $3 x=5$ the value of $x$ in each of the four equations is different suggesting that $x$ is a generalized number which represents different numbers depending on the equation given. Philipp (1999), is in support with the concept of a variable as a generalized number and added that a variable can take many values in certain situations.

The study has revealed that there are misconceptions in algebra among grade 11 learners in the two selected schools of the study. The misconceptions could be attributed to learners' lack of understanding of algebraic concepts which also can be traced to lack of arithmetic skills. Algebra is regarded as generalized arithmetic, 
suggesting that procedures and rules found in arithmetic can be applied to algebra. The difference between the two being that arithmetic only uses numbers, while algebra uses both numbers and letters. Letters are called variables or generalized numbers. This is because they can represent different numbers depending on the context. It is therefore recommended that teachers should ensure that learners have a sound understanding of arithmetic concepts before teaching algebra. Concepts such as additive and multiplicative inverses should be taught so that they can find their application in solving equations

\subsection{REFERENCES}

Ausubel, D. P. (1976). Psychology of Education. America.

Cohen, L., Manion, L., Morrison K. (2000) Research Methods in Education ( $5^{\text {th }}$ ed.) London: RoutledgeFalmer

Eggen, P. D., \& Kauchak, D. P. (2000). Educational psychology: Windows on classrooms (5 ${ }^{\text {th }}$ ed.). Upper Saddle River, NJ: Prentice Hall.

Gunawardena, E. (2011). Secondary School Students' Misconception in algebra. University of Toronto.

Maguire. J. T. (2012). Investigation of Misconceptions Related to the Concepts of Equivalence and Literal Symbols Held by Underprepared Community College Students. San Francisco University.

Mwape (2016). Mathematics Teacher Education curriculum at a University in Zambia: Student Teachers' Acquisition of Appropriate competencies for Teaching Mathematics in Secondary School.

Philipp, R. A. (1999). The many uses of algebraic variables. In Moses (Ed), Algebraic thinking, Grades K-12: Readings from the NCTM's school- based journals and other publications (pp. 157-162). Reston, VA: National Council of Teachers of Mathematics.

Piaget, J. (1970). Cognitive development. London.

Skemp, R. (1971). The Psychology of learning mathematics. Penguin books. 